Check for updates

Cite this: RSC Adv., 2018, 8, 32461

Received 3rd August 2018

Accepted 11th September 2018

DOI: $10.1039 / c 8 r a 06543 h$

rsc.li/rsc-advances

\section{Degradation of recalcitrant organics in landfill concentrated leachate by a microwave-activated peroxydisulfate process}

\author{
Zhepei Gu, ${ }^{a}$ Weiming Chen, ${ }^{\mathrm{b}}$ Qibin Li, ${ }^{\mathrm{b}}$ Ying Wang, ${ }^{\mathrm{a}}$ Chuanwei Wu \\ and Aiping Zhang (D) *a
}

A microwave (MW)-activated peroxydisulfate (PDS) process was applied to remove recalcitrant organics in concentrated leachate. In this study, the optimum activation conditions were studied using the absorbance at $254 \mathrm{~nm}\left(\mathrm{UV}_{254}\right)$ and color number removal efficiencies and by comparison of the different processes. The inner oxidation mechanism was investigated by ultraviolet-visible (UV-vis) spectrophotometry and threedimensional (3D) excitation-emission matrix (EEM) tests. The results show that oxidation effects followed the order MW/PDS > MW/ $\mathrm{H}_{2} \mathrm{O}_{2}>$ heat/PDS. The efficiencies of chemical oxygen demand (COD) removal, $U_{254}$, and color number were $45.50 \%, 48.95 \%$, and $88.35 \%$, respectively. The biodegradability was enhanced to 0.23 under optimum conditions (initial $\mathrm{pH}$ of $3, \mathrm{MW}$ irradiation power of $450 \mathrm{~W}$, PDS dosage of $3.5 \mathrm{~g} \mathrm{~L}^{-1}$, and reaction time of $10 \mathrm{~min}$ ). The UV-vis spectra suggest that the humification degree and aromaticity of organics in the concentrated leachate greatly declined in the MW/PDS process. 3D EEM spectra indicate that the molecular weight of organic substances in the concentrated leachate decreased markedly and that the constitution of the organics became simpler after the MW/ PDS process. In a word, the MW/PDS process is a promising method for concentrated leachate treatment.

\section{Introduction}

Large quantities of leachates are produced by decomposition of municipal solid waste (MSW) in landfills by factors such as rainfall and runoff. Leachates have complex composition and are typically heavily contaminated, very hazardous, and difficult to treat. ${ }^{\mathbf{1 , 2}}$ Many organic pollutants in leachate are included in USEPA's list of priority pollutants. Leachate that is treated improperly can lead to heavy pollution of the surrounding water and soil. Reverse osmosis (RO) and nanofiltration (NF), a method combining a biological process and membrane technology, is capable of removing almost all of the organic pollutants in leachates, but it has the main drawback of producing concentrated leachate at proportions of $0.5-30 \% .^{3,4}$ Concentrated leachate contains a high concentration of recalcitrant macromolecular organics, hazardous organics, and residual metal ions; ${ }^{5}$ in addition, its high concentration of salt would be intercepted by the membrane. Hence, efficient treatment using a biological process is difficult to achieve.

\footnotetext{
${ }^{a}$ Key Laboratory of Treatment for Special Wastewater of Sichuan Province Higher Education System, College of Chemistry and Materials Science, Sichuan Normal University, Chengdu 610066, China. E-mail: apzhang@sicnu.edu.cn; Tel: +86 13488951126

${ }^{b}$ Faculty of Geosciences and Environmental Engineering, Southwest Jiaotong University, Chengdu 611765, China
}

At present, common treatment technologies for concentrated leachate include recirculation, concentration, coagulation, and advanced oxidation processes (AOPs). The cost of recirculation is low, and this method can accelerate the stabilization of MSW, but it is not capable of effectively degrading recalcitrant organics and removing salts. ${ }^{6,7}$ Additionally, leachate treatment is influenced by the production effluent and the quality of RO and NF technology. ${ }^{8}$ Membrane technology can effectively decrease the $\mathrm{COD}, \mathrm{BOD}_{5}$, and total nitrogen content; ${ }^{9-11}$ however, it is constrained by the limited service life of RO and NF membranes and by the increasing treatment expense due to membrane pollution by extremely high concentrations of humic acid and fulvic acid. ${ }^{\mathbf{1 2}}$ Coagulation is widely applied to the pretreatment of concentrated leachate because of its convenient operation and its low cost; however, it is not very effective in removing organics in concentrated leachate. ${ }^{\mathbf{1 3}}$ AOPs play an important role in water treatment, ${ }^{\mathbf{1 4 - 1 7}}$ utilizing produced radicals with strong oxidizing ability, $(\cdot \mathrm{OH}$ and $\mathrm{SO}_{4}{ }^{-}$), thus effectively degrading organics in concentrated leachate. ${ }^{\mathbf{1 8 , 1 9}}$ Although AOPs have many advantages such as high reaction rate and high treatment efficiency, the high cost of their processing has mainly constrained their application. Fenton and ozone method are the most common AOPs. The conventional Fenton method is limited by its low reaction rate, strict $\mathrm{pH}$ condition, low utilization of $\mathrm{H}_{2} \mathrm{O}_{2}$, and production of iron sludge. ${ }^{20,21}$ The ozone method is limited by the high 
production cost of ozone and its low utilization. Moreover, the treatment efficiency of single AOPs is quite limited.

Persulfate (as peroxymonosulfate or peroxydisulfate, PDS) is well known for its stable capacity and high water solubility. It has attracted much attention as a Fenton-like reagent in wastewater treatment since the publication of studies of Anipsitakis and Dionysiou. ${ }^{22,23}$ Persulfate has low oxidizing capacity $\left(E^{0}=2.01 \mathrm{~V}\right)$, but after activation by factors such as such as heat, ultraviolet radiation, ultrasound, microwave (MW) (eqn (1)), and transition metals (eqn (2)), ${ }^{23-27}$ it can generate sulfate radical $\left(\mathrm{SO}_{4}{ }^{-}\right)$. This radical has a high oxidation potential, i.e., $2.6 \mathrm{~V}$, which is close to that of hydroxyl radical $\left(E^{0}=2.8 \mathrm{~V}\right.$, halflife $<1 \mathrm{~s}$ ). It also has a long half-life, i.e., $4 \mathrm{s,}{ }^{28,29}$ which enables it to oxidize recalcitrant organics at high efficiency. Many activation methods use much energy, thus increasing the treatment cost, and transition-metal activation leads to secondary pollution due to metal corrosion and metal recycling. ${ }^{30}$ Thus, an economic and efficient activation method for persulfate activation is urgently needed.

$$
\begin{gathered}
\mathrm{S}_{2} \mathrm{O}_{8}{ }^{2-} \stackrel{\text { heat/irradiation }}{\longrightarrow} 2 \mathrm{SO}_{4}{ }^{-} \\
\mathrm{S}_{2} \mathrm{O}_{8}^{2-}+\mathrm{M}^{n+} \rightarrow \mathrm{SO}_{4}{ }^{--}+\mathrm{SO}_{4}^{2-}+\mathrm{M}^{n+1}
\end{gathered}
$$

MW is an electromagnetic wave with a frequency of $300 \mathrm{MHz}$ to $300 \mathrm{GHz}$ and has both thermal and non-thermal effects. In contrast to conventional heating, MW heating can cause dipolar molecules to rotate and collide and thus raise them to an excited state, which increases the collision possibility and reduces the reaction time. ${ }^{31}$ Because of the thermal and nonthermal effects of MW irradiation, persulfate can produce sulfate radical, which can effectively degrade acid orange $7,{ }^{32}$ reactive yellow $145,{ }^{33}$ sulfamethoxazole, ${ }^{34}$ and pentachlorophenol $^{35}$ in aqueous solution. In particular, MW can substantially increase the reaction rate as compared with conventional heating. ${ }^{36} \mathrm{Kim}^{37}$ systematically studied the decomposition of landfill leachate by persulfate under MW assistance and proved that this process is capable of removing organics from landfill leachate. However, few works have investigated the application of the MW/PDS process as a pretreatment for concentrated leachate. Few also have compared the oxidation efficiencies and degradation mechanisms of the heat/PDS, MW/ $\mathrm{H}_{2} \mathrm{O}_{2}$, and MW/ PDS processes.

In the present study, we aimed (1) to study the effects of persulfate dosage, MW irradiation power, and initial $\mathrm{pH}$ in the process on the degradation efficiency of recalcitrant organics; (2) to compare different processes for concentrated leachate treatment; and (3) to investigate the oxidation mechanism in each process and compare them using ultraviolet-visible (UVvis) spectra and three-dimensional excitation-emission matrix (3D EEM) spectra.

\section{Materials and methods}

\subsection{Concentrated leachate samples}

Concentrated leachate samples were collected from a landfill in southwestern China that adopts a technology with the

\begin{tabular}{|c|c|c|c|c|c|c|}
\hline Index & $\begin{array}{l}\text { COD } \\
(\mathrm{mg} / \mathrm{L})\end{array}$ & $\begin{array}{l}\mathrm{UV}_{254} \\
\left(\mathrm{~cm}^{-1}\right)\end{array}$ & $\begin{array}{l}\mathrm{CN}^{a} \\
\left(\mathrm{~cm}^{-1}\right)\end{array}$ & $\mathrm{pH}$ & $\begin{array}{l}\text { BOD } \\
(\mathrm{mg} / \mathrm{L})\end{array}$ & $\mathrm{B} / \mathrm{C}$ \\
\hline Value & 1690 & 6.170 & 3.350 & 7.81 & 17 & 0.01 \\
\hline
\end{tabular}

Table 1 Characteristics of the concentrated leachate

combination process of anoxic oxic (A/O), anoxic oxic $(\mathrm{A} / \mathrm{O})$, membrane bioreactor (MBR), and nanofiltration (NF) or reverse osmosis (RO). The daily capacity of membrane filtration for the concentrated leachate is $200 \mathrm{~m}^{3} \mathrm{~d}^{-1}$. Each concentrated leachate's color was originally dark brown, and it had no obvious stench. Characteristics of the concentrated leachate are listed in Table 1. According to the 3D EEM spectrum, the fluorescence index (f450/500) is 1.61, showing that the concentrated leachate was mainly terrigenous and of biological origin. Its humification index (8.96) shows that the humification degree is high; its biological source index (0.92) indicates that it originated from an autogenous medium. In all, the collected concentrated leachate has typical characteristics of a recalcitrant pollutant.

\subsection{Reagents}

Potassium persulfate $\left(\mathrm{K}_{2} \mathrm{~S}_{2} \mathrm{O}_{8}\right)$, hydrogen peroxide $\left(\mathrm{H}_{2} \mathrm{O}_{2}\right)$, sodium hydroxide $(\mathrm{NaOH})$, and other reagents, all of analytical grade, were purchased from Kelong Co. Ltd., Chengdu (China). The MW irradiation initiator (M1-211A) was from Midea Co. Ltd. (China).

\subsection{Experimental procedure}

First, $100 \mathrm{~mL}$ of concentrated leachate ( $\mathrm{pH}$ adjusted using $\mathrm{NaOH}$ and $\mathrm{H}_{2} \mathrm{SO}_{2}$ ) was transferred to a $250 \mathrm{~mL}$ round-bottom flask. A preset dosage of PDS was then added, and the flask was placed in the MW initiator. After a preset reaction time, the MW oven was switched off, and the flask was cooled in ice water. After the sample was cooled, it was filtered through 0.45 $\mu \mathrm{m}$ glass fiber filters and then water testing was conducted.

\subsection{Analytical methods}

The COD of samples was determined by MW rapid digestion titration method, in accordance with the Water QualityDetermination of the Chemical Oxygen Demand-Dichromate Method (HJ 828-2017). ${ }^{38}$ BOD $_{5}$ was determined by following the Water Quality-Determination of Biochemical Oxygen Demand after 5 Days $\left(\mathrm{BOD}_{5}\right)$ for Dilution and Seeding Method (HJ 505-2009). ${ }^{38}$ The absorbance of the sample at $254 \mathrm{~nm}\left(\mathrm{UV}_{254}\right)$ was analyzed to ascertain the amount of humic acid. The color number was evaluated from that the absorbances of sample at 436,525 , and $620 \mathrm{~nm}\left(A_{436}, A_{525}\right.$, and $A_{620}$ respectively; eqn (3)) in order to determine the content of humic substance in the concentrated leachate. The COD removal efficiency was calculated using eqn (4):

$$
\mathrm{CN}=\frac{A_{436}{ }^{2}+A_{525}{ }^{2}+A_{620}{ }^{2}}{A_{436}+A_{525}+A_{620}} .
$$



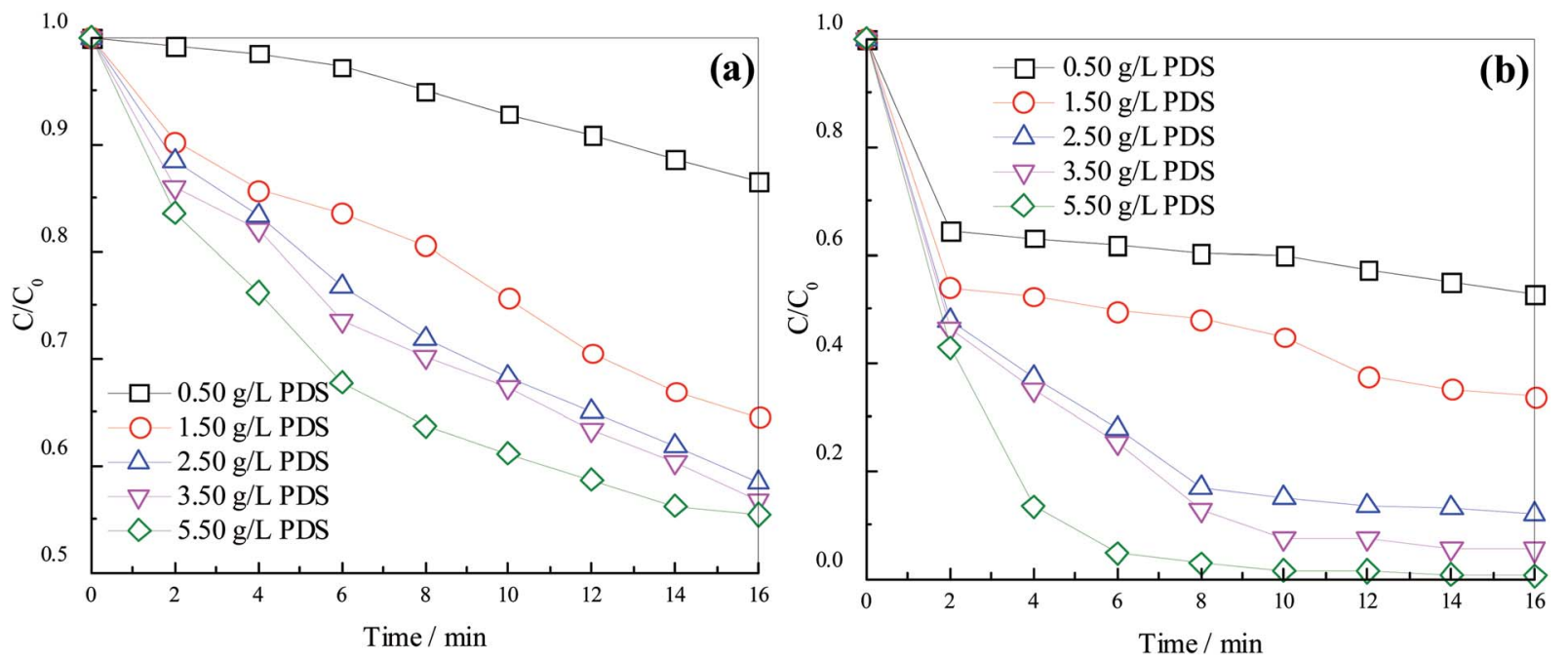

Fig. 1 Effects of PDS dosage on the degradation of concentrated leachate at an MW irradiation power of $450 \mathrm{~W}$ and PDS dosages of $0.50,1.50$, $2.50,3.50$, and $5.50 \mathrm{~g} \mathrm{~L}^{-1}$ : (a) $\mathrm{UV}_{254}$ and (b) color number. Conditions: initial pH of $3, \mathrm{MW}$ irradiation power of $450 \mathrm{~W}$, and reaction time of 10 min.

$$
\text { COD removal }(\%)=\frac{\mathrm{COD}_{\mathrm{i}}-\mathrm{COD}_{\mathrm{t}}}{\mathrm{COD}_{\mathrm{t}}} \times 100
$$

where $\mathrm{COD}_{\mathrm{i}}$ and $\mathrm{COD}_{\mathrm{t}}$ are the $\mathrm{COD}\left(\mathrm{mg} \mathrm{L}^{-1}\right)$ of the concentrated samples before and after each process.

UV-vis spectrometry using $1 \mathrm{~cm}$ quartz cells was applied to record the absorbances of samples from 220 to $800 \mathrm{~nm}$ and to reveal the degradation extent of organics in the concentrated leachate. The sample was filtered through a $0.45 \mu \mathrm{m}$ glass fiber filter after elimination of residual hydrogen peroxide and then diluted with ultrapure water obtained by secondary reverse osmosis. A Perkin-Elmer Lambda 950 UV-vis spectrometer (USA) was used. The test wavenumber range was $220-600 \mathrm{~nm}$, and the scan interval was $1 \mathrm{~nm}$.

3D EEM spectra were used to characterize the aromatic dissolved organic matter (DOM) in concentrated leachate. Synchronized adsorption was observed using 3D fluorescence spectra obtained with a Horiba Scientific Aqualog-UV-800-C (USA). The excitation wavelength slit width was $5 \mathrm{~nm}$, the scan speed was $500 \mathrm{~nm} \mathrm{~min}{ }^{-1}$, the excitation wavelength was $239-550 \mathrm{~nm}$, and the emission wavelength was $230-650 \mathrm{~nm}$. 3D EEM spectra were obtained using a CCD detector. Ultrapure water was used to obtain the blank value, and Rayleigh scattering and Raman scattering were eliminated by using a Horiba Scientific software kit. Spectra were constructed and the isoheight was set using Origin 9.0 software.

\section{Results and discussion}

\subsection{Effects of PDS dosage on the efficiency of concentrated leachate degradation}

On the basis of the MW activation process, the effects of PDS dosage on degradation efficiency of concentrated leachate were investigated using the $\mathrm{UV}_{254}$ and color number. Fig. 1 shows the degradation efficiencies of concentrated leachate.

As shown in Fig. 1, $\mathrm{UV}_{254}$ and color number decreased with the reaction time in the process, indicating that an increasing dosage of PDS enhanced the degradation of organics. Removal efficiencies as indicated by $\mathrm{UV}_{254}$ and color number increased from $13.45 \%$ and $47.35 \%$ to $44.57 \%$ and $94.63 \%$, respectively, when the reaction time was $16 \mathrm{~min}$ and as the PDS dosage increased from $0.50 \mathrm{~g} \mathrm{~L}^{-1}$ to $5.50 \mathrm{~g} \mathrm{~L}^{-1}$. The results indicate that more active species formed with the increase in PDS dosage, thus strengthening the oxidizing ability and accelerating the degradation of organics, consistent with the conclusion of Qi. $^{34,36}$ At a reaction time of $16 \mathrm{~min}$, removal efficiencies of $\mathrm{UV}_{254}$ and color number increased by $28.20 \%$ and $40.73 \%$, respectively, when the PDS dosage increased from 0.50 to $2.50 \mathrm{~g} \mathrm{~L}^{-1}$. Further increase in PDS dosage to 3.50 and $5.50 \mathrm{~g} \mathrm{~L}^{-1}$ apparently did not increase the removal efficiencies. We can see that a PDS dosage of $3.50 \mathrm{~g} \mathrm{~L} \mathrm{~L}^{-1}$ was optimal, considering the economical aspect; the removal rate first increased, and finally tended to decrease more slowly under this condition. In the early stage of reaction, MW can pyrolyze organics and destroy their chemical bonds; ${ }^{31}$ meanwhile, PDS can undergo activation, producing sulfate radical, which oxidizes organics in wastewater. In the later stage of reaction, the concentration of organics decreased, thus stopping reaction despite the increase in sulfate radical concentration. On the other hand, the excessive PDS can quench sulfate radical (eqn (5)). A similar process was reported by Hori. ${ }^{39}$ Sulfate radical is also a scavenger for itself (eqn (6)), resulting in consumption of a large amount of oxidized matter and the weakening effect of sulfate radical due its decreasing quantity. However, the removal efficiency was relatively stable because of the compensation of thermal and non-thermal effects of MW with the decrease in radicals concentration. $^{40}$

$$
\begin{gathered}
\mathrm{SO}_{4}{ }^{--}+\mathrm{S}_{2} \mathrm{O}_{8}^{2-} \leftrightarrow \mathrm{SO}_{4}^{2-}+\mathrm{S}_{2} \mathrm{O}_{8}^{\cdot-} \\
\mathrm{SO}_{4}{ }^{\cdot-}+\mathrm{SO}_{4}{ }^{--} \rightarrow \mathrm{S}_{2} \mathrm{O}_{8}^{2-}
\end{gathered}
$$




\subsection{Effects of $\mathrm{pH}$ on the degradation of concentrated leachate}

The species and quantity of radicals are related to the $\mathrm{pH}$. Sulfate radical is dominant under acidic conditions, and hydroxyl radical is dominant under neutral and alkaline conditions (Liang, 2009). Hence, the effects of $\mathrm{pH}(2,3,4,6$, and 10) on the degradation efficiency in the process were studied (results are shown in Fig. 2).

Different $\mathrm{pH}$ levels had large impacts on the removal of organics, as shown in Fig. 2. Clearly, acidic conditions enhanced the removal. At a reaction time of $16 \mathrm{~min}$, the $\mathrm{UV}_{254}$ and color number increased from $18.96 \%$ and $42.06 \%$ to $44.41 \%$ and $98.31 \%$, respectively, when the $\mathrm{pH}$ decreased from 10 to 2 . This result can be explained as follows. First, persulfate has a certain oxidation capacity $\left(E^{0}=2.01 \mathrm{~V}\right)$ that can lead to production of sulfate radical $\left(E^{0}=2.60 \mathrm{~V}\right)$ by MW activation (eqn (1)). Second, these reactions can take place under acidic conditions (eqn (7) and (8)), increasing the production of sulfate radical and thus enhancing the $\mathrm{UV}_{254}$ and color number. Third, sulfate radical can react with $\mathrm{OH}^{-}$to form hydroxyl radical under alkaline conditions (eqn (9)). However, sulfate radical (half-life $=4 \mathrm{~s}$ ) has stability better than that of hydroxyl radical (half-life of $<1 \mathrm{~s}$; Nosaka, 2002), and it has the same oxidation capacity as hydroxyl radical under acidic conditions. ${ }^{23}$ Thus, the generation of hydroxyl radical under alkaline conditions reduced the amount of sulfate radical to a certain degree, and low $\mathrm{pH}$ showed a positive effect on $\mathrm{UV}_{254}$ and color number. Moreover, when the $\mathrm{pH}$ decreased from 10 (alkaline condition) to 6 (acid condition) and the reaction time reached $16 \mathrm{~min}$, the $\mathrm{UV}_{254}$ and color number were remarkably enhanced. The $\mathrm{UV}_{254}$ did not change as much as that of color number; this may because radicals in the process first react with chromophores, decompose them into micromolecular organics, and then continuously react with radicals.

$$
\begin{gathered}
\mathrm{S}_{2} \mathrm{O}_{8}^{2-}+\mathrm{H}^{+} \rightarrow \mathrm{HS}_{2} \mathrm{O}_{8}^{-\cdot} \\
\mathrm{HS}_{2} \mathrm{O}_{8}^{-} \rightarrow \mathrm{SO}_{4}^{\cdot-}+\mathrm{SO}_{4}^{2-}+\mathrm{H}^{+} \\
\mathrm{SO}_{4}^{\cdot-}+\mathrm{OH}^{-} \rightarrow \mathrm{SO}_{4}^{2-}+\cdot \mathrm{OH}
\end{gathered}
$$

\subsection{Effects of MW irradiation power on the degradation of concentrated leachate}

Sulfate radical can be produced from PDS by MW activation. The production of sulfate radical is vital to the degradation of organics in concentrated leachate. The effects of different MW irradiation powers $(100,300,450$, and $800 \mathrm{~W})$ on $\mathrm{UV}_{254}$ and color number are shown in Fig. 3.

As illustrated in Fig. 3, the removal efficiency increased with the MW power. At a reaction time of $16 \mathrm{~min}, \mathrm{UV}_{254}$ and color number increased from $19.45 \%$ and $51.56 \%$ to $54.94 \%$ and $96.89 \%$, respectively, when the MW irradiation power increased from 100 to $800 \mathrm{~W}$. The temperature of the sample rose faster with rising MW irradiation power, and the quantity of sulfate radical increased because of the thermal effect of MW irradiation. On the other hand, an increase in MW irradiation power enhanced the reactivity of dipolar molecules, which absorbed the MW irradiation, and the probability of collision between molecules, thus increasing the reaction rate. Meanwhile, the non-thermal effect of MW irradiation can decrease the reaction activation energy, further shortening the reaction time. Removal efficiencies at 450 and $800 \mathrm{~W}$ were similar, implying that MW irradiation power reached optimum condition. In addition, the removal efficiency before 10 min was much higher than that after $10 \mathrm{~min}$. This can be explained by the increased production of sulfate radical and oxidation rate due to the gradual rise of process temperature and the increase in the amount of sulfate radical. ${ }^{33}$ Before $10 \mathrm{~min}$, a large amount of
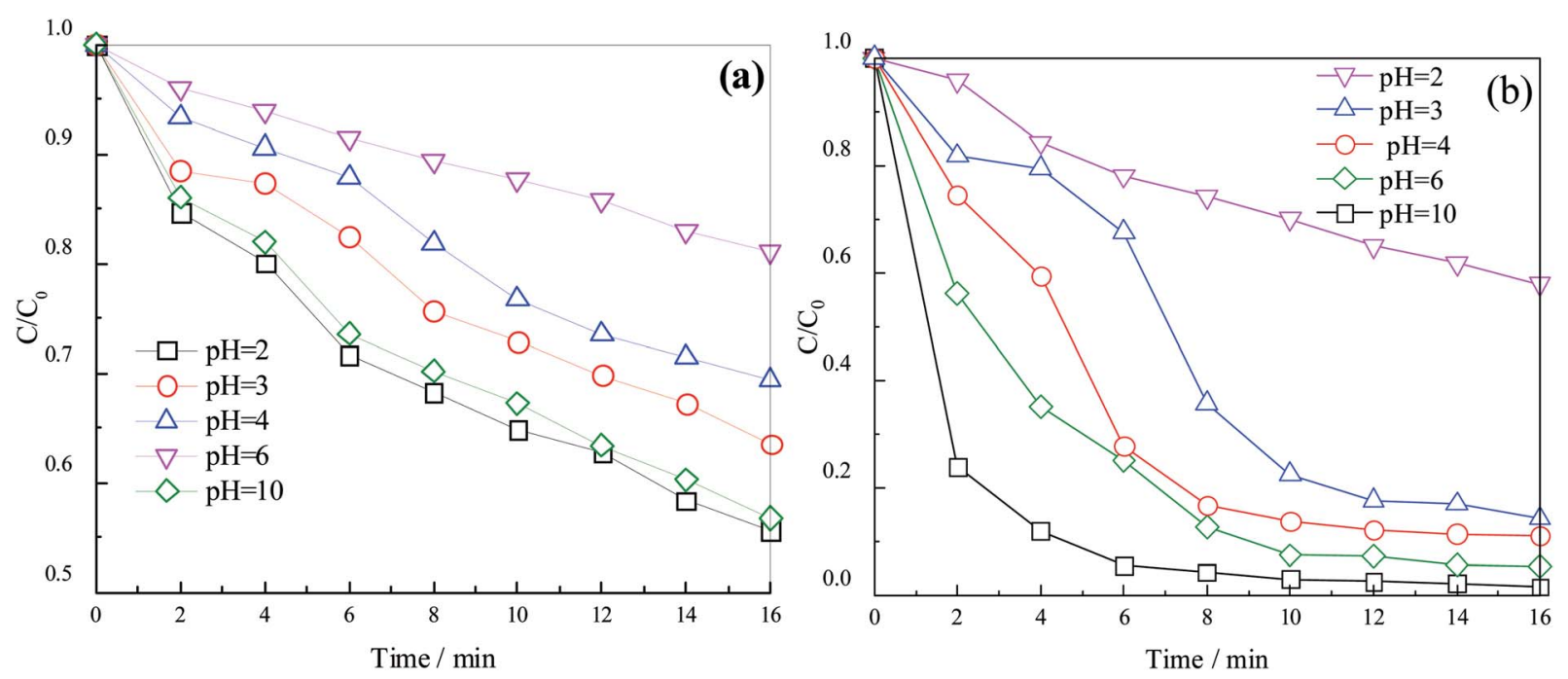

Fig. 2 Effects of $\mathrm{pH}\left(2,3,4,6\right.$, and 10) on the degradation of concentrated leachate at an MW power of $450 \mathrm{~W}$ and a PDS dosage of $3.50 \mathrm{~g} \mathrm{~L}{ }^{-1}$ : (a) $\mathrm{UV}_{254}$ and (b) color number. Conditions: MW irradiation power of $450 \mathrm{~W}$, PDS dosage of $3.5 \mathrm{~g} \mathrm{~L}^{-1}$, and reaction time of $10 \mathrm{~min}$. 

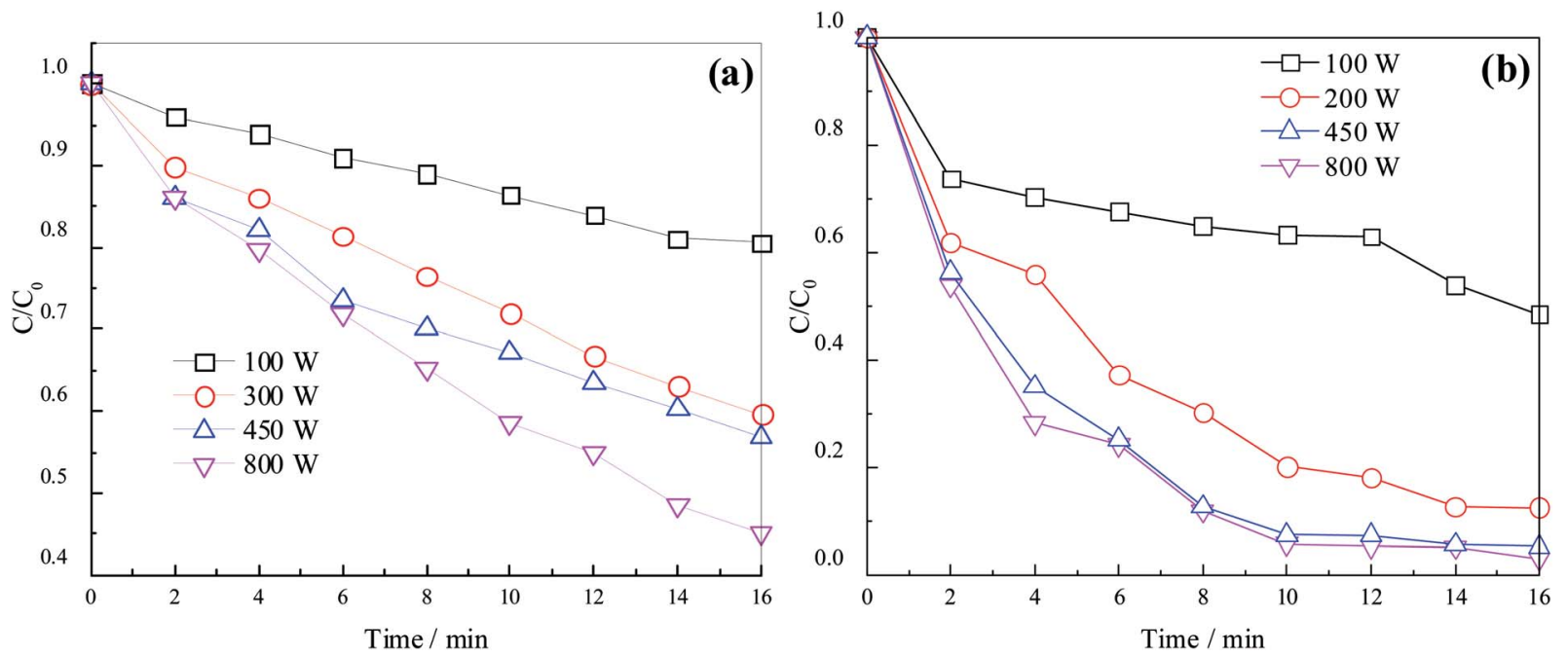

Fig. 3 Effects of MW irradiation power $\left(100,300,450\right.$, and $800 \mathrm{~W}$ ) on the degradation of concentrated leachate at a PDS dosage of $3.50 \mathrm{~g} \mathrm{~L}{ }^{-1}$ : (a) $\mathrm{UV}_{254}$ and (b) color number. Conditions: initial $\mathrm{pH}$ of 3, MW irradiation power of $450 \mathrm{~W}$, and PDS dosage of $3.5 \mathrm{~g} \mathrm{~L}^{-1}$.

sulfate radical could oxidize most organics effectively; after $10 \mathrm{~min}$, most organics were degraded, and changes in the removal efficiency were not obvious. On the basis of the above observations, the optimum conditions were an MW irradiation of $450 \mathrm{~W}$ and a reaction time of $10 \mathrm{~min}$.

\subsection{Comparison of different processes and analysis of biodegradability}

The characteristics of different processes on the degradation of organics at a temperature of $85{ }^{\circ} \mathrm{C}$, MW irradiation power of $450 \mathrm{~W}$, PDS dosage of $3.5 \mathrm{~g} \mathrm{~L}^{-1}$, and $\mathrm{H}_{2} \mathrm{O}_{2}$ dosage of $0.24 \mathrm{~g} \mathrm{~L}^{-1}$ are shown in Fig. 4.

As shown in Fig. 4, the removal efficiency of the MW/PDS process was much higher than those of the other processes. The removal efficiencies of conventional heat and single MW processes were $1.75 \%$ and $2.99 \%$ (COD), $2.59 \%$ and $4.05 \%$ $\left(\mathrm{UV}_{254}\right)$, and $18.41 \%$ and $21.23 \%$ (color number), respectively; in addition, biodegradability in both processes was extremely low (heat, 0.01 and $\mathrm{MW}, 0.02$ ). In the absence of oxidizing reagents, COD removal efficiencies, $\mathrm{UV}_{254}$, and color number were low; however, the MW process results were better than those of the heat process. Heat alone can volatilize some of the organics with low boiling point, resulting in low removal efficiency. On the other hand, the thermal and non-thermal effects of MW irradiation can destroy unstable chemical bonds with increasing process temperature, thus giving good results. However, conventional heat and single MW processes did not have an obvious effect on the organic composition of wastewater on a macroscopic level.

In the PDS, heat/PDS, and MW/PDS processes, the removal efficiencies were $8.37 \%, 27.46 \%$, and $45.50 \%$ (COD); $10.37 \%$, $34.36 \%$, and $48.95 \%\left(\mathrm{UV}_{254}\right)$; and $38.73 \%, 47.39 \%$, and $86.35 \%$ (color number), respectively. Additionally, the biodegradability of the samples after the processes were 0.08 (PDS), 0.18 (heat/ PDS), and 0.23 (MW/PDS), indicating that the MW/PDS process can effectively degrade recalcitrant organics in wastewater and enhance the biodegradability remarkably. In the presence of an oxidizing reagent (PDS, $E^{0}=2.01 \mathrm{~V}$ ), the PDS process had better removal efficiencies than those of conventional heat and MW processes. Both the heat/PDS and MW/PDS process produced sulfate radical $\left(E^{0}=2.6 \mathrm{~V}\right)$ and gave good results, but the MW/PDS process had better removal efficiency. MW activated the PDS process, which produced sulfate radical. On the other hand, MW irradiation is more effective than heat because MW irradiation can cause dipolar molecules to rotate and vibrate intensely, increasing the probability of contact between organic molecules and oxidation reagents. To sum up, the MW/PDS process can produce radicals, resulting in high efficiency of removal of organics in wastewater.

In the $\mathrm{MW} / \mathrm{H}_{2} \mathrm{O}_{2}$ and MW/PDS processes, the removal efficiencies were $41.12 \%$ and $45.50 \%$ (COD), $41.00 \%$ and $48.95 \%$ $\left(\mathrm{UV}_{254}\right)$, and $73.49 \%$ and $88.35 \%$ (color number), respectively. Both processes could effectively decrease the COD of wastewater and enhance the biodegradability to $0.21\left(\mathrm{MW} / \mathrm{H}_{2} \mathrm{O}_{2}\right)$ and 0.23 (MW/PDS). Overall, the MW/PDS process had better results. MW activated $\mathrm{H}_{2} \mathrm{O}_{2}$, producing hydroxyl radical $\left(E^{0}=2.8 \mathrm{~V}\right)$, which can effectively oxidize organics in wastewater and enhance biodegradability. But extension of the reaction time caused mineralization of organics and a decrease in $\mathrm{pH}$ in the process, resulting in reduction of $\mathrm{H}_{2} \mathrm{O}_{2}$ to $\mathrm{H}_{2} \mathrm{O}$ and decreasing the production of hydroxyl radical. On the other hand, PDS can produce a large amount of sulfate radical by MW activation with increasing temperature and declining pH (eqn (7) and (8)), as reported in studies of Qi. ${ }^{34,41}$ Thus, the MW/PDS process can remarkably enhance the wastewater treatment efficiency.

\subsection{UV-vis spectra analysis of the different processes}

The absorbance in the UV-vis spectra was related to the species and concentration of the organics in wastewater. In the ultraviolet region (200-380 nm), the absorbance was related to the complexity and amount of aromatic compounds in wastewater. To analyze the degradation characteristics in the different 

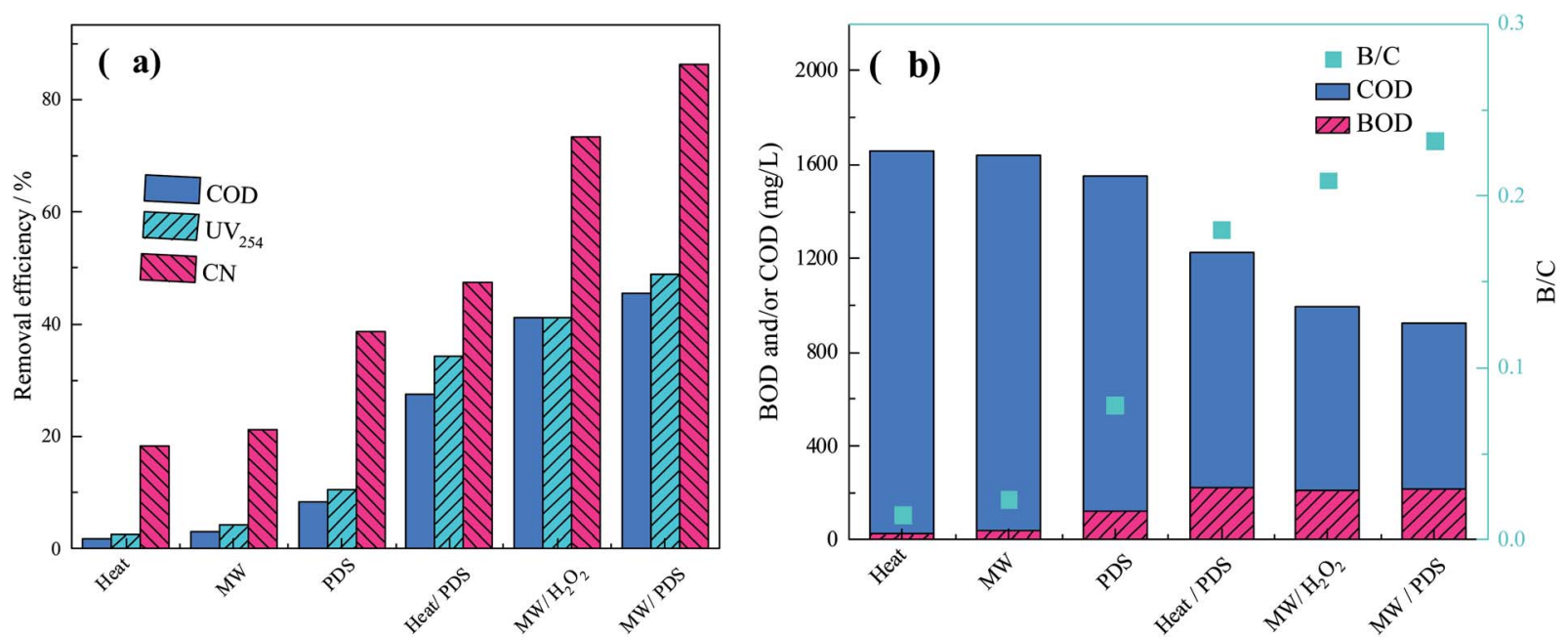

Fig. 4 Removal efficiencies of different processes on (a) COD, UV 254 , and color number and (b) biodegradability. Conditions: (1) reaction temperature of $85^{\circ} \mathrm{C}$ for the heat process, (2) MW power of $450 \mathrm{~W}$ for the MW process, (3) PDS dosage of $3.5 \mathrm{~g} \mathrm{~L}^{-1}$ for the PDS process, (4) reaction temperature of $85^{\circ} \mathrm{C}$ and PDS dosage of $3.5 \mathrm{~g} \mathrm{~L}^{-1}$ for the heat/PDS process, (5) MW power of $450 \mathrm{~W}^{2}$ and $\mathrm{H}_{2} \mathrm{O}_{2}$ dosage of $0.24 \mathrm{~g} \mathrm{~L}-1$ for the $\mathrm{MW} / \mathrm{H}_{2} \mathrm{O}_{2}$ process, and (6) $\mathrm{MW}$ power of $450 \mathrm{~W}$ and PDS dosage of $3.5 \mathrm{~g} \mathrm{~L}^{-1}$ for the MW/PDS process. Reaction times were both 15 min.

processes, the variation of dissolved organic matter was investigated. The UV-vis spectra from the different processes and the specific absorbance values are shown in Fig. 5 .

As demonstrated in Fig. 5 , the absorbance was related to the concentration of organics; however, the absorbance decreased to different extents after each process. It is worth noting that the absorbances for the heat, MW, and PDS processes were higher than those of raw concentrated leachate. This result can be explained by the absence of oxidants in the heat and MW processes; both have thermal effects and water may evaporate as the reaction time increases, resulting in an increment of the concentration of organics. The complexity of the PDS process might increase because of the addition of oxidants; however, the oxidation effect was not good because there was no activation. Hence, the incomplete oxidation might result in the production of many relatively small molecules and aromatic substances. Overall, the absorbances follow the trend heat $>$ MW $>$ PDS $>$ raw concentrated leachate. However, a significant decrease occurred in the heat/PDS, $\mathrm{MW} / \mathrm{H}_{2} \mathrm{O}_{2}$, and MW/PDS processes, especially, in the MW/PDS process, suggesting that the three processes can destroy the molecular structure of organics and decrease the aromaticity and complexity of wastewater.

Both $E_{254}$ and $E_{280}$ (absorbance at 254 and $280 \mathrm{~nm}$, respectively) represent the aromaticity of organics in concentrated leachate. ${ }^{2,42,43}$ In addition, a significant peak at
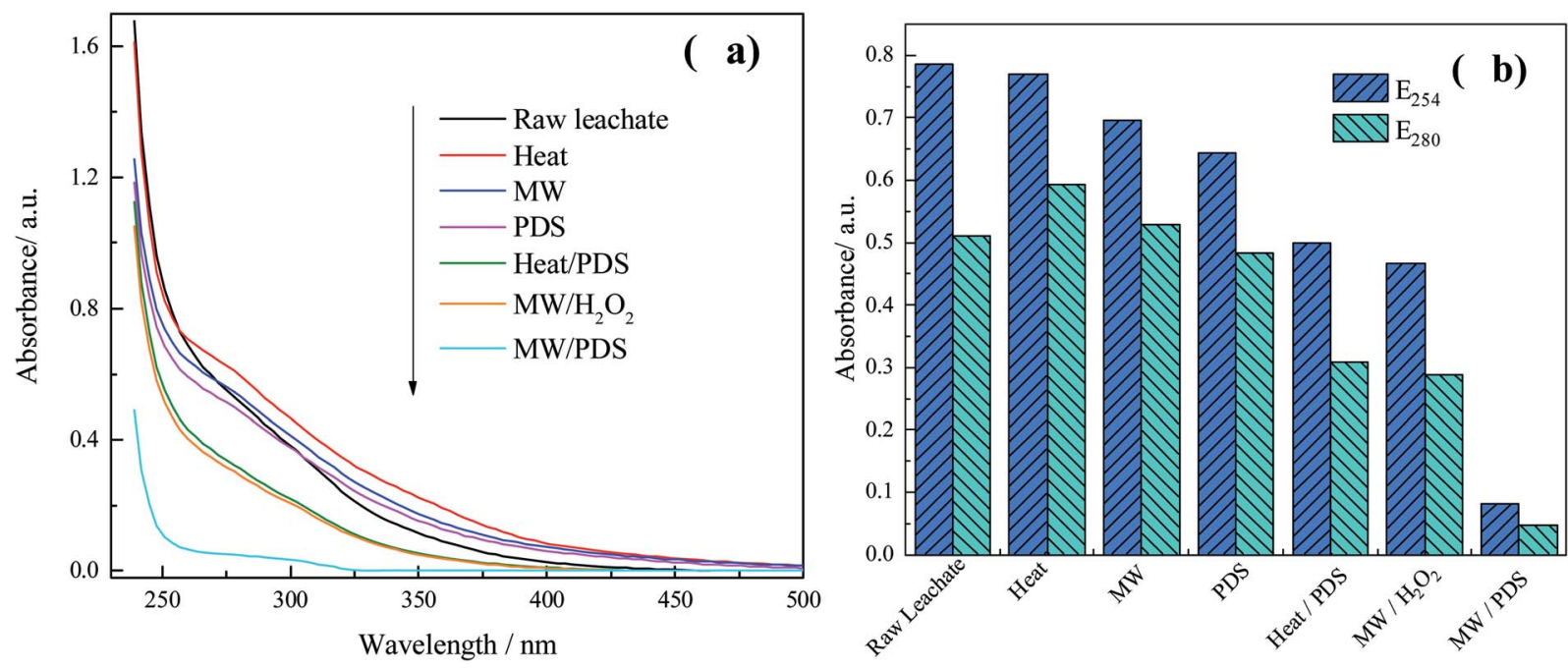

Fig. 5 UV-vis spectra: (a) absorbance spectra for samples and (b) specific indexes of $E_{254}$ and $E_{280}$ after the different processes. Conditions: (1) reaction temperature of $85^{\circ} \mathrm{C}$ for the heat process, (2) MW power of $450 \mathrm{~W}$ for the MW process, (3) PDS dosage of $3.5 \mathrm{~g} \mathrm{~L}^{-1}$ for the PDS process, (4) reaction temperature of $85^{\circ} \mathrm{C}$ and PDS dosage of $3.5 \mathrm{~g} \mathrm{~L}^{-1}$ for the heat/PDS process, (5) MW power of $450 \mathrm{~W}^{2}$ and $\mathrm{H}_{2} \mathrm{O}_{2}$ dosage of $0.24 \mathrm{~g} \mathrm{~L}^{-1}$ for the $\mathrm{MW} / \mathrm{H}_{2} \mathrm{O}_{2}$ process, and (6) MW power of $450 \mathrm{~W}$ and PDS dosage of $3.5 \mathrm{~g} \mathrm{~L}^{-1}$ for the MW/PDS process. Both reaction times were 15 min. 

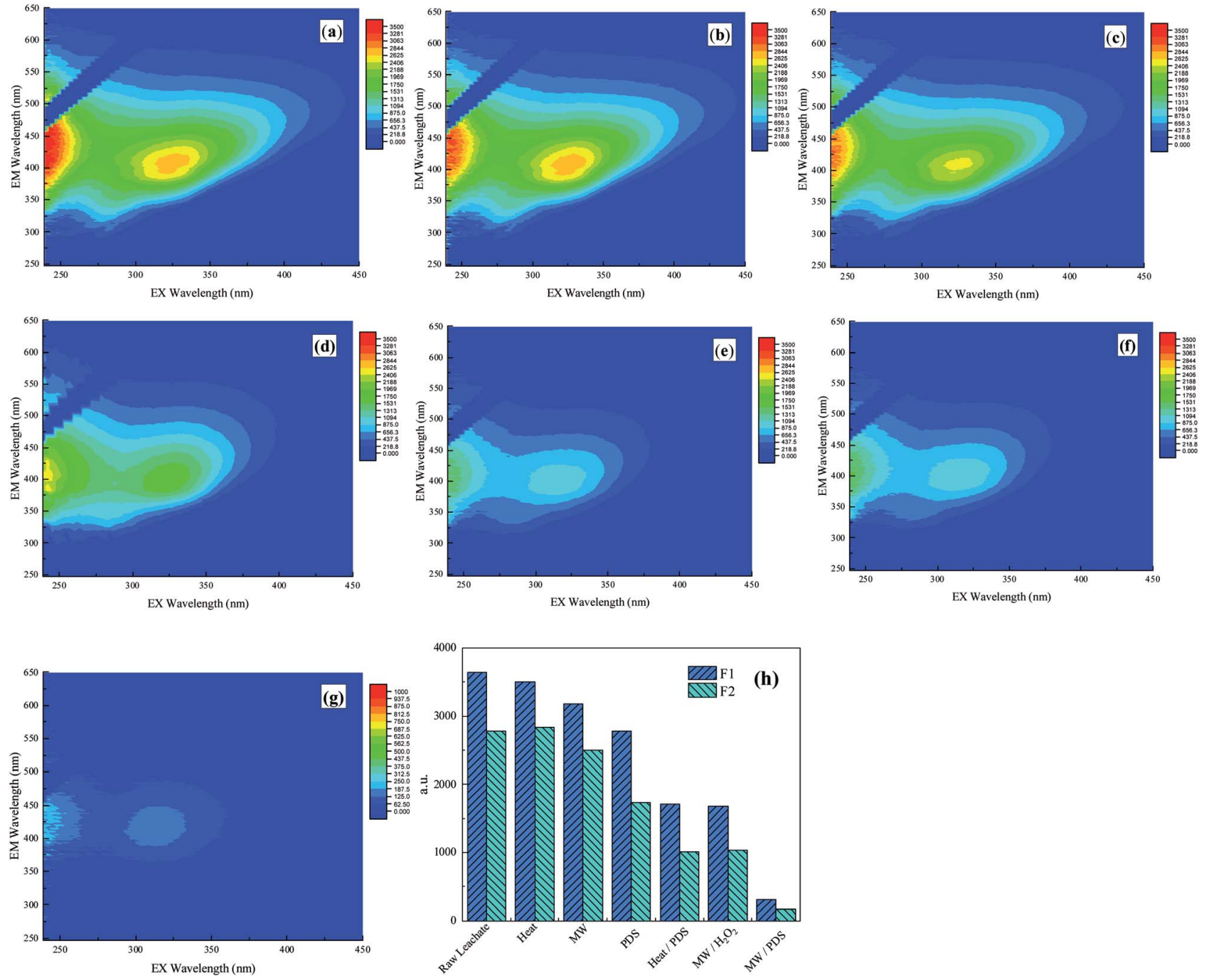

Fig. 6 3D EEM spectra of concentrated leachate before and after different processes: (a) raw concentrated leachate, (b) heat process, (c) MW process, (d) PDS process, (e) heat/PDS process, (f) $\mathrm{MW} / \mathrm{H}_{2} \mathrm{O}_{2}$ process, and (g) MW/PDS process. (h) Peak values of $\mathrm{F} 1$ and $\mathrm{F} 2$ in each process. Conditions: initial $\mathrm{pH}$ of 3, MW irradiation power of $450 \mathrm{~W}$, PDS dosage of $3.5 \mathrm{~g} \mathrm{~L}^{-1}$, reaction temperature of $85^{\circ} \mathrm{C}_{,} \mathrm{H}_{2} \mathrm{O}_{2}$ dosage of $0.24 \mathrm{~g} \mathrm{~L}-1$, and reaction time of $10 \mathrm{~min}$.

220-250 $\mathrm{nm}$ indicates that the organics in concentrated leachate contain conjugated and unsaturated bonds. ${ }^{44} \mathrm{~A}$ visible absorbance peak in the $250-290 \mathrm{~nm}$ range shows that concentrated leachate contained aromatic heterocyclic rings. ${ }^{2}$ Moreover, the absorbance at $290-350 \mathrm{~nm}$ shows that the DOM had carbonyl groups, conjugated groups, or both. ${ }^{45}$ $E_{254}$ and $E_{280}$ in the MW/PDS process sharply decreased from 0.79 and 0.51 to 0.08 and 0.04 , respectively, revealing that the aromaticity of organics in the concentrated leachate declined substantially. In addition, each absorption value in the different processes (heat/PDS, $\mathrm{MW} / \mathrm{H}_{2} \mathrm{O}_{2}$, and MW/PDS processes) greatly decreased. This shows that these processes can effectively destroy the structure of recalcitrant organics in concentrated leachate, leading to a tendency for a simple constitution of the concentrated leachate. Overall, the results obtained in the UV-vis analysis are consistent with the COD and biodegradability.

\subsection{D EEM spectrum analysis of samples before and after the different processes}

3D EEM spectra were widely used to characterize the dissolved organic matter and to determine the fluorescence index, humification degree, and molecular weight. ${ }^{46-49}$ To investigate the removal efficiencies of the humic substances in the different processes, 3D EEM spectra of the samples after the different processes are compared and illustrated in Fig. 6 . In the spectrum, F1 $\left(\lambda_{\mathrm{Ex}} / \lambda_{\mathrm{Em}}=310-360 \mathrm{~nm} / 370-450 \mathrm{~nm}\right)$ represents substances resembling fulvic acid in the visible region, which are mainly relative stable, and macromolecular aromatic substances. ${ }^{49,50} \mathrm{~F} 2\left(\lambda_{\mathrm{Ex}} / \lambda_{\mathrm{Em}}=235-255 \mathrm{~nm} / 410-450 \mathrm{~nm}\right)$ represents the substances resembling fulvic acid in the ultraviolet region, which are mainly small-molecular-weight and highfluorescence-intensity matter. ${ }^{51-54}$ The above results show that the concentrated leachate contained a large amount of recalcitrant organics. 
As shown in Fig. 6, the fluorescence peak values of raw concentrated leachate were 3467.86 (F1) and 2775.07 (F2), and each fluorescence peak value was different. In the different processes, the fluorescence peak values of substances resembling fulvic acid in the ultraviolet and visible regions decreased after each process. In the conventional heat and MW process, the removal efficiencies at the fluorescence peak values were low because of the absence of oxidants; however, a better result was observed in the MW process because of the non-thermal effect. In the PDS, heat/ PDS, and MW/PDS processes, the values of F1 and F2 decreased to $2780.67,1709.99$, and 312.15 (F1) and to $1735.87,1012.92$, and 164.78 (F2), respectively. These results reveal that MW can effectively activate PDS and enhance the oxidizing ability of the process. Comparison of the $\mathrm{MW} / \mathrm{H}_{2} \mathrm{O}_{2}$ and MW/PDS processes showed that the peak values of both F1 and F2 decreased significantly and that the removal efficiencies were $52.03 \%$ and $63.48 \%\left(\mathrm{MW} / \mathrm{H}_{2} \mathrm{O}_{2}\right)$ and $91.09 \%$ and $94.17 \%$ (MW/PDS), respectively. These findings suggest that these processes were capable of degrading substances resembling fulvic acid in both the ultraviolet and visible regions, and that the MW/PDS process was more effective in the degradation of organics than was the $\mathrm{MW} / \mathrm{H}_{2} \mathrm{O}_{2}$ process. After MW/PDS treatment, the fluorescence peak of raw concentrated leachate shifted from $239 \mathrm{~nm}$ and $454 \mathrm{~nm}$ and from $326 \mathrm{~nm}$ and $407 \mathrm{~nm}$ to $239 \mathrm{~nm}$ or $447 \mathrm{~nm}$ and $410 \mathrm{~nm}$, respectively, indicating that the molecular weight and humification degree declined remarkably and that the constitution of organics tended to be relatively simple after oxidation in the process. ${ }^{55,56}$

\section{Conclusions}

In this study, MW-activated PDS process proved to be a promising method for the treatment of concentrated leachate. By investigating the effects of PDS dosage, $\mathrm{pH}$ change, and MW irradiation power, the COD removal efficiencies, $\mathrm{UV}_{254}$, and color number were found to be $45.50 \%, 48.95 \%$, and $88.35 \%$, respectively. The biodegradability was markedly enhanced to 0.23 at a PDS dosage of $3.5 \mathrm{~g} \mathrm{~L}^{-1}$, an MW irradiation power of $450 \mathrm{~W}$, and a reaction time of $16 \mathrm{~min}$. The, MW/PDS process proved to be the most efficient process in enhancing the effluent quality among the different treatment processes. The UV-vis spectra suggest that the humification degree of concentrated leachate decreased and that the constitution of organics in the concentrated leachate tended to be simple. By analyzing 3D EEM spectra, we found that the concentration of substances resembling fulvic acid in concentrated leachate decreased substantially, that the humification degree declined, and that the recalcitrant macromolecular organics decomposed to relatively simple small-molecule organic substances.

\section{Conflicts of interest}

There are no conflicts to declare.

\section{Acknowledgements}

The authors gratefully acknowledge the financial support of the Key Laboratory of Treatment for Special Wastewater (SWWT2015-4).

\section{References}

1 A. Imai, T. Fukushima, K. Matsushige, Y. H. Kim and K. Choi, Water Res., 2002, 36, 859-870.

2 K. H. Kang, H. S. Shin and H. Park, Water Res., 2002, 36, 4023-4032.

3 D. Dolar, K. Košutić and T. Strmecky, Sep. Purif. Technol, 2016, 168, 39-46.

4 J. Huang, J. Chen, Z. Xie and X. Xu, Environ. Technol., 2015, 36, 1001-1007.

5 J. L. de Morais and P. P. Zamora, J. Hazard. Mater., 2005, 123, 181-186.

6 H. D. Robinson and P. J. Maris, Water Res., 1983, 17, 15371548.

7 I. A. Talalaj and P. Biedka, Ecol. Eng., 2015, 85, 185-192.

8 H. Wang, Y. N. Wang, X. Li, Y. Sun, H. Wu and D. Chen, J. Waste Manage., 2016, 56, 271-279.

9 Z. Li, S. Zhou and J. Qiu, Environ. Eng. Sci., 2007, 24, 12451256.

10 W. Rukapan, B. Khananthai, T. Srisukphun, W. Chiemchaisri and C. Chiemchaisri, Water Sci. Technol., 2015, 71, 580.

11 Y. Zhou, M. Huang, Q. Deng and T. Cai, Desalination, 2017, 420, 99-105.

12 Q. Q. Zhang, B. H. Tian, X. Zhang, A. Ghulam, C. R. Fang and R. He, J. Waste Manage., 2013, 33, 2277-2286.

13 Y. Long, J. Xu, D. Shen, Y. Du and H. Feng, Chemosphere, 2017, 167, 512-519.

14 F. Chen, Q. Yang, Y. Zhong, H. An, J. Zhao, T. Xie, Q. Xu, X. Li, D. Wang and G. Zeng, Water Res., 2016, 101, 555-563.

15 F. Chen, Q. Yang, X. Li, G. Zeng, D. Wang, C. Niu, J. Zhao, H. An, T. Xie and Y. Deng, Appl. Catal., B, 2017, 200, 330-342.

16 F. Chen, Q. Yang, S. Wang, F. Yao, J. Sun, Y. Wang, C. Zhang, X. Li, C. Niu, D. Wang and G. Zeng, Appl. Catal., B, 2017, 209, 493-505.

17 F. Chen, Q. Yang, J. Sun, F. Yao, S. Wang, Y. Wang, X. Wang, X. Li, C. Niu, D. Wang and G. Zeng, ACS Appl. Mater. Interfaces, 2016, 8, 32887-32900.

18 A. Fernandes, L. Labiadh, L. Ciríaco, M. J. Pacheco, A. Gadri, S. Ammar and A. Lopes, Chemosphere, 2017, 184, 1223.

19 X. Jing, Y. Long, D. Shen, H. Feng and T. Chen, J. Hazard. Mater., 2017, 323, 674-680.

20 A. A. Babaei, B. Kakavandi, M. Rafiee, F. Kalantarhormizi, I. Purkaram, E. Ahmadi and S. Esmaeili, J. Ind. Eng. Chem., 2017, 56, 163-174.

21 D. Hermosilla, M. Cortijo and C. P. Huang, Sci. Total Environ., 2009, 407, 3473-3481.

22 G. P. Anipsitakis and D. D. Dionysiou, Environ. Sci. Technol., 2003, 37, 4790.

23 G. P. Anipsitakis and D. D. Dionysiou, Environ. Sci. Technol., 2004, 38, 3705. 
24 F. Ghanbari and M. Moradi, Chem. Eng. J., 2017, 102, 307315.

25 P. Hu and M. Long, Appl. Catal., B, 2016, 181, 103-117.

26 Y. B. Kim and J. H. Ahn, Korean J. Chem. Eng., 2017, 34, 19801984.

27 Q. Yang, Y. Zhong, H. Zhong, X. Li, W. Du, X. Li, R. Chen and G. Zeng, Process Saf. Environ. Prot., 2015, 98, 268-275.

28 Y. Nosaka, M. Nakamura and T. Hirakawa, Phys. Chem. Chem. Phys., 2002, 4, 1088-1092.

29 X. H. Xia, J. L. Xu and Y. Yun, J. Environ. Sci., 2002, 14, 188194.

30 Z. Li, Q. Yang, Y. Zhong, X. Li, L. Zhou, X. Li and G. Zeng, RSC Adv., 2015, 6, 987-994.

31 C. Costa, V. H. S. Santos, P. H. H. Araujo, C. Sayer, A. F. Santos and M. Fortuny, Eur. Polym. J., 2009, 45, 20112016.

32 S. Yang, P. Wang, X. Yang, G. Wei, W. Zhang and L. Shan, J. Environ. Sci., 2009, 21, 1175-1180.

33 N. N. Patil and S. R. Shukla, Journal of Water Process Engineering, 2015, 7, 314-327.

34 C. Qi, X. Liu, C. Lin, X. Zhang, J. Ma, H. Tan and W. Ye, Chem. Eng. J., 2014, 249, 6-14.

35 C. Qi, X. Liu, W. Zhao, C. Lin, J. Ma, W. Shi, Q. Sun and H. Xiao, Environ. Sci. Pollut. Res. Int., 2015, 22, 4670-4679.

36 C. Qi, X. Liu, C. Lin, H. Zhang, X. Li and J. Ma, Chem. Eng. J., 2017, 315, 201-209.

37 Y. B. Kim and J. H. Ahn, J. Environ. Eng., 2016, 142, 04015084.

38 W. Chen, A. Zhang, Z. Gu and Q. Li, Chem. Eng. J., 2018, 354, 680-691.

39 H. Hori, A. Yamamoto, E. Hayakawa, S. Taniyasu, A. Nobuyoshi Yamashita, S. Kutsuna, H. K. And and R. Arakawa, Environ. Sci. Technol., 2005, 39, 2383-2388.
40 X.-Y. Yu, Z.-C. Bao and J. R. Barker, ChemInform, 2004, 35, 295-308.

41 C. Qi, X. Liu, C. Lin, H. Zhang, X. Li and J. Ma, Chem. Eng. J., 2017, 315, 201-209.

42 G. V. Korshin, M. M. Benjamin and R. S. Sletten, Water Res., 1997, 31, 1643-1650.

43 F. Lü, H. Zhang, C. H. Chang, D. J. Lee, P. J. He, L. M. Shao and A. Su, Chemosphere, 2008, 72, 1381-1386.

44 B. Lai, Y. Zhou, J. Wang, Z. Yang and Z. Chen, Chemosphere, 2013, 93, 2805-2813.

45 Y. P. Chin, G. Aiken and E. O'Loughlin, Environ. Sci. Technol., 1994, 28, 1853-1858.

46 A. Baker and M. Curry, Water Res., 2004, 38, 2605-2613.

47 L. Guo, M. Lu, Q. Li, J. Zhang, Y. Zong and Z. She, Bioresour. Technol., 2014, 171, 22-28.

48 J. A. Leenheer and J. P. Croué, Environ. Sci. Technol., 2003, 37, 18A-26A.

49 W. Chen, P. Westerhoff, J. A. Leenheer and K. Booksh, Environ. Sci. Technol., 2015, 37, 5701-5710.

50 C. A. Stedmon and S. Markager, Limnol. Oceanogr., 2005, 50, 686-697.

51 X. S. He, B. D. Xi, X. Li, H. W. Pan, D. An, S. G. Bai, D. Li and D. Y. Cui, Chemosphere, 2013, 93, 2208-2215.

52 X. S. He, B. D. Xi, R. T. Gao, H. Zhang, Q. L. Dang, D. Li and C. H. Huang, Chemosphere, 2016, 144, 75-80.

53 F. Lu, C. H. Chang, D. J. Lee, P. J. He, L. M. Shao and A. Su, Chemosphere, 2009, 74, 575-582.

54 X. Wang, F. Zhang, H. T. Kung, A. Ghulam, A. L. Trumbo, J. Yang, Y. Ren and Y. Jing, Catena, 2017, 155, 62-74.

55 Z. P. Liu, W. H. Wu, P. Shi, J. S. Guo and J. Cheng, J. Waste Manage., 2015, 41, 111-118.

56 H. Wang, Y. Wang, Z. Lou, N. Zhu and H. Yuan, J. Waste Manage., 2017, 69, 274-280. 